\title{
Application of Immunocytochemistry and BRAF Mutational Analysis to Direct Smears of Metastatic Melanoma
}

Kim Hookim, MD*, Michael H. Roh, MD, PhD*, Joseph Willman, MD, Jeremiah Placido, MD, Helmut C. Weigelin, MLS (ASCP), Kristina L. Fields, BS, Judy Pang, MD, Bryan L. Betz, PhD; and Stewart M. Knoepp, MD, PhD

BACKGROUND: The cytodiagnosis of melanoma in fine-needle aspiration (FNA) specimens can be challenging, often requiring the use of immunocytochemistry. As constitutively activating mutations in the BRAF oncogene are present in at least $40 \%$ of melanomas, the use of FNA material to interrogate the $B R A F$ mutational status is likely to increase. Because cell blocks, traditionally used for these studies, can occasionally exhibit insufficient tumor cellularity, the authors investigated the utility of direct smears for immunocytochemistry and BRAF mutational analysis. METHODS: Immunocytochemistry for S-10O, HMB-45, and Mart-1 was prospectively performed on direct smears in 17 FNAs of metastatic melanoma. Next, BRAF sequencing was performed using DNA isolated from archived Diff-Quik-stained direct smears for 15 cases. In parallel, sequencing was performed using DNA obtained from corresponding cell blocks. RESULTS: S100 positivity in the tumor cells was observed in all 17 cases. HMB-45 and Mart-1 positivity was noted in $81 \%$ and $88 \%$ of cases, respectively. All 3 markers were positive in $76 \%$ of cases. Next, of the 15 archived melanoma FNAs tested, BRAF mutations were observed in 8 (53\%); 5 and 3 melanomas harbored the V6OOE and V600K mutation, respectively. Corresponding cell blocks were also tested for all 15 cases, yielding concordant BRAF results in 14 (93\%); 1 cell block yielded a false-negative result. CONCLUSIONS: Cytologic direct smears represent a robust and valuable source of cellular material for immunocytochemistry and molecular studies, especially in instances in which inadequate cell block cellularity is anticipated or encountered. Cancer (Cancer Cytopathol) 2012;120:52-61. (c) 2011 American Cancer Society.

KEY WORDS: melanoma, BRAF, immunocytochemistry, direct smear, cytology, S-100, HMB-45, Mart-1.

In 2010 , it is estimated that $>68,000$ men and women were diagnosed with melanoma and 8700 died of the disease. ${ }^{1}$ The morbidity and mortality associated with melanoma stems from a multitude of factors including challenges in establishing a diagnosis, limited understanding of the molecular pathogenesis underlying the disease, unpredictable presentation of metastatic foci, and limited treatment options for those with advanced disease. ${ }^{2,3}$ Surgical therapy is more effective in patients with localized or early melanoma; however, melanoma that has spread to locoregional lymph nodes or distant sites is largely refractory

Corresponding author: Stewart M. Knoepp, MD, PhD, Department of Pathology, University of Michigan Health System, 1500 E. Medical Center Dr, Ann Arbor, MI 48109; Fax: (734) 763-4095; sknoepp@med.umich.edu

*The first 2 authors contributed equally to this work.

Department of Pathology, University of Michigan Medical School, Ann Arbor, Michigan

Received: April 19, 2011; Revised: June 6, 2011; Accepted: June 8, 2011

Published online July 25, 2011 in Wiley Online Library (wileyonlinelibrary.com)

DOI: $10.1002 /$ cncy.20180, wileyonlinelibrary.com 
to currently available systemic therapies such as high-dose interferon, dacarbazine, temozolomide, and high-dose interleukin-2; the survival advantage offered by these agents is marginal. $^{2}$

Fine-needle aspiration (FNA) represents a minimally invasive technique and an accurate, safe, and affordable means to achieve a tissue diagnosis. FNA is therefore becoming an increasingly used modality to establish a diagnosis of metastatic melanoma. ${ }^{3-6}$ An early definitive diagnosis of metastatic melanoma can facilitate prompt, appropriate management including surgical removal or avoidance of unnecessary surgery and accurate staging of patients in clinical trials. ${ }^{6}$

Because melanoma can exhibit a variety of cytomorphologic features and mimic other neoplasms such as carcinomas and sarcomas, identifying melanoma in aspirates and distinguishing it from its mimics can occasionally be challenging. ${ }^{7-9}$ The presence of melanin pigment can be helpful in the cytodiagnosis of melanoma; nonetheless, amelanotic melanomas are commonly encountered in FNAs. Specifically, through their analysis of a large series of melanoma FNAs, Saqi et al ${ }^{9}$ discovered that $68 \%$ of melanoma specimens failed to demonstrate any identifiable intracytoplasmic melanin pigment. Hence, cytopathologists can potentially face difficult scenarios in which a melanoma must be accurately distinguished from benign, reactive processes and from other malignant entities based on limited material. Immunocytochemistry is commonly used to confirm a diagnosis of melanoma in FNA specimens. ${ }^{8,10-13}$ Common markers used in this regard are antibodies directed against S-100, HMB-45, and Mart-1/ Melan-A.

In addition, the lack in understanding of signature genetic aberrations associated with melanoma has confounded the ability for targeted therapy for metastatic melanoma. However, the recent discovery that constitutively activating mutations in the $B R A F$ oncogene are present in at least $40 \%$ of melanomas has created strategic opportunities for targeted therapeutics. ${ }^{2,14,15}$ The V600E and $\mathrm{V} 600 \mathrm{~K}$ substitutions represent the 2 most common $B R A F$ mutations in melanoma. ${ }^{16}$ Clinical trials are currently being performed to assess the efficacy of BRAF inhibitors in the treatment of melanoma. Recently, Flaherty et $\mathrm{al}^{2}$ reported that in patients with melanomas harboring the BRAF V600E mutation, therapy with PLX4032 results in partial tumor regression in the major- ity of cases. Another report by Rubinstein et $\mathrm{al}^{17}$ suggests that melanomas harboring the V600K mutation could also respond to PLX4032.

Routinely, immunocytochemical and molecular ancillary studies have used cell blocks prepared from melanoma FNAs. Occasionally, insufficient cellularity of the cell blocks can be a problematic issue, thereby impeding the performance of these assays. This can lead to repeat procedures to obtain additional cellular material, which are not without potential complications. Given the inherent unpredictability of cell block cellularity and the increased need to interrogate the immunophenotype and $B R A F$ mutation status of melanoma FNAs, the purpose of this study was 2-fold. We first sought to prospectively investigate the application of immunocytochemistry for S-100, HMB-45, and Mart-1 to cytologic direct smears. We then extended our analysis by demonstrating that $B R A F$ mutation testing can be effectively performed on Diff-Quik-stained cytologic smears.

\section{MATERIALS AND METHODS}

This study was approved by the Institutional Review Board at the University of Michigan. First, 17 consecutive cases of metastatic melanoma were prospectively evaluated in which unstained direct smears were prepared, using positively charged slides, for confirmatory immunocytochemistry. Immunocytochemistry was performed on air-dried, unstained direct smears after fixation in formalin for 30 minutes using the Ventana Autostainer (Ventana Medical Systems, Tucson, Ariz) as performed previously. ${ }^{18}$ Incubation with a rabbit polyclonal antibody directed against S-100 (prediluted; Ventana Medical Systems) was performed for 24 minutes without pretreatment. Incubation with mouse monoclonal antibodies against HMB-45 (prediluted; Dako, Carpenteria, Calif) and Mart-1 (clone A103, prediluted; Ventana Medical Systems) were performed for 16 minutes and 20 minutes after antigen retrieval with $\mathrm{CC} 1$ buffer $(\mathrm{pH} 8.5)$ at $95^{\circ} \mathrm{C}$ for 8 minutes and 36 minutes, respectively. Positive and negative controls were performed in parallel. Negative controls were performed on unstained direct smears. Positive controls were performed using formalin-fixed, paraffin-embedded sections of melanoma. Furthermore, for comparison, cell block sections were used for immunohistochemistry when tumor cells were present in the cell 
block (14 of 17 cases). The cell blocks in 3 cases were acellular, precluding comparative immunohistochemistry. Sixteen cases of metastatic melanoma were amelanotic and 3,3'-diaminobenzidine (DAB) was used as the chromogen. In the remaining case, abundant melanin pigment was present; hence, immunostaining using alkaline phosphatase was used. Cytoplasmic and/or nuclear staining for S-100 and cytoplasmic staining for HMB-45 and Mart- 1 were scored semiquantitatively by 3 cytopathologists (K.H., M.H.R., and S.M.K.) using a 3-tier scoring system in which 0 represented negative staining and $1+$, $2+$, and $3+$ corresponded to immunoreactivity in $<$ $10 \%, 10 \%$ to $50 \%$, and $>50 \%$ of the tumor cells, respectively.

Next, air-dried, Diff-Quik-stained smears from 15 consecutive FNAs of metastatic melanoma (performed between January 2010 and July 2010) in which at least 2 Diff-Quik-stained smears with abundant diagnostic material were present were retrieved from the archive and selected for BRAF mutation testing. Diff-Quik-stained smears (1 slide per case) were incubated in xylene for 1 week. After the coverslips were gently removed, the slides were allowed to dry, reviewed by 2 cytopathologists (K.H. and M.H.R.), and the area most enriched in tumor cells was marked on the underside of each slide using a marking pen. The size of the selected area (in $\mathrm{mm}^{2}$ ) and the percentage of tumor cells within the area were recorded. In addition, the corresponding cytologic cell block was retrieved for each case for parallel $B R A F$ mutation testing. The cellularity of each cell block was semiquantitatively assessed by evaluating the original hematoxylin and eosin (H \& E)-stained cell block sections as follows: 0, acellular; $1+$, sparse cellularity; $2+$, moderate cellularity; and $3+$, abundant cellularity. The estimated percentage of tumor cells was also recorded for each cell block section.

For the Diff-Quik-stained direct smears, genomic DNA was extracted from the marked region using the Pinpoint Slide DNA Isolation Kit (Zymo Research Corporation, Orange, Calif) as per the manufacturer's instructions and including the optional purification step. Briefly, Pinpoint Solution was applied to the designated area of each slide to be extracted and allowed to air dry into a thin film. The film was then gently lifted and transferred to a $1.5-\mathrm{mL}$ microcentrifuge tube, and $50 \mu \mathrm{L}$ of 1-step Extraction Buffer containing proteinase $\mathrm{K}$ was added and incubated at $55^{\circ} \mathrm{C}$ for 4 hours, followed by incubation at $95^{\circ} \mathrm{C}$ for
10 minutes. An optional DNA cleanup step was performed for each sample by adding $100 \mu \mathrm{L}$ of Pinpoint Binding Buffer to each proteinase K-treated DNA sample and then transferring the solution to a supplied spin column. The spin column was microcentrifuged for $10 \mathrm{sec}-$ onds at 14,000g and washed twice with Pinpoint Wash Buffer with microcentrifugation between washes, followed by a final centrifugation step $(14,000 \mathrm{~g}$ for 1 minute). DNA was eluted in a final volume of $25 \mu \mathrm{L}$ of TE buffer $(10 \mathrm{mM}$ of Tris- $\mathrm{HCl}$ and $0.5 \mathrm{mM}$ of ethylenediamine tetraacetic acid [EDTA] [pH 9.0]). For paraffin-embedded cytology cell blocks, genomic DNA was extracted on the BioRobot EZ1 (Qiagen, Chatsworth, Calif) using the paraffin section protocol. For each block, 5 sections measuring $10 \mu \mathrm{m}$ in thickness were used for extraction. DNA was eluted in a final volume of $100 \mu \mathrm{L}$ of TE buffer.

$B R A F$ mutation status was evaluated by direct sequencing. A 204-base pair fragment containing $B R A F$ exon 15 was amplified using the following primer pairs: 5'TGCTTGCTCTGATAGGAAAATG3' (forward) and 5'TCAGTGGAAAAATAGCCTCAATTC3' (reverse). Each $30-\mu \mathrm{L}$ polymerase chain reaction (PCR) contained 5 $\mu \mathrm{L}$ of purified DNA, $300 \mathrm{nM}$ of each primer, and $1 \times$ Phusion HF mastermix (Finnzymes Thermo Scientific, Pittsburgh, Pa). Cycling conditions were comprised of denaturation at $98^{\circ} \mathrm{C}$ for 30 seconds followed by 40 amplification cycles: $99^{\circ} \mathrm{C}$ for 5 seconds, $60^{\circ} \mathrm{C}$ for $20 \mathrm{sec}-$ onds, and $72^{\circ} \mathrm{C}$ for 20 seconds. An aliquot of each PCR product was confirmed by gel electrophoresis. The remainder was purified by the QIAquick PCR purification kit (Qiagen) and subjected to bidirectional sequencing with ABI BigDye v1.1 terminators and the following

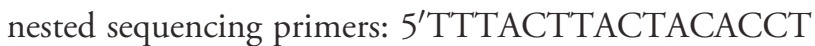
CAGATAT3' (forward) and 5'GGAAAAATAGCCTCA ATTCTTACC3' (reverse). Sequence products were purified using the DyeEx Spin Kit (Qiagen) and analyzed on the ABI 3130xl genetic analyzer (Applied Biosystems, Bedford, Mass). Both forward and reverse sequence chromatograms were reviewed for mutations with softwareassisted analysis (Mutation Surveyor, SoftGenetics, St. College, Pa).

\section{RESULTS}

Immunocytochemistry for S-100, HMB-45, and Mart-1 was prospectively performed on unstained, air-dried 
Table 1. Immunocytochemistry on Direct Smears of Metastatic Melanoma

\begin{tabular}{|c|c|c|c|c|}
\hline \multirow[t]{2}{*}{ Case No. } & \multirow[t]{2}{*}{ FNA Site } & \multicolumn{3}{|c|}{$\begin{array}{c}\text { Results of } \\
\text { Immunocytochemistry }^{a}\end{array}$} \\
\hline & & S-100 & HMB-45 & Mart-1 \\
\hline 1 & Left groin & $3+$ & NP & $1+$ \\
\hline 2 & Left neck & $3+$ & $3+$ & $3+$ \\
\hline 3 & Right groin & $3+$ & $2+$ & $3+$ \\
\hline 4 & Right axillary lymph node & $3+$ & $3+$ & $3+$ \\
\hline 5 & Right chest & $3+$ & $2+$ & $3+$ \\
\hline 6 & Left shoulder & $3+$ & 0 & $2+$ \\
\hline 7 & Right posterior thigh & $3+$ & $1+$ & $3+$ \\
\hline 8 & Subcarinal lymph node & $3+$ & $3+$ & $3+$ \\
\hline 9 & Right dorsal foot & $3+$ & $2+$ & $3+$ \\
\hline 10 & Left chest & $3+$ & 0 & 0 \\
\hline 11 & Left neck & $1+$ & 0 & 0 \\
\hline 12 & Left neck & $3+$ & $3+$ & $3+$ \\
\hline 13 & Right hilar lymph node & $3+$ & $3+$ & $3+$ \\
\hline 14 & Right supraclavicular lymph node & $2+$ & $2+$ & $3+$ \\
\hline 15 & Right axillary lymph node & $3+$ & $3+$ & $3+$ \\
\hline 16 & Left inguinal lymph node & $3+$ & $1+$ & $3+$ \\
\hline 17 & Subcarinal lymph node & $3+$ & $2+$ & $3+$ \\
\hline
\end{tabular}

\footnotetext{
Abbreviations: FNA, fine-needle aspiration; NP, not performed.

${ }^{\text {a } A}$ score of 0 denotes negative staining in all of the tumor cells. Scores of $1+, 2+$, and $3+$ denote immunoreactivity in $<10 \%, 10 \%$ to $50 \%$, and $>50 \%$ of the tumor cells, respectively.
}

direct smears for 17 FNAs of metastatic melanoma. All the patients had a history of melanoma. The FNA sites and the results of immunocytochemistry are summarized in Table 1. No significant intracytoplasmic melanin pigment was appreciated in the tumor cells in 16 of the 17 cases. In all 17 cases, immunoreactivity of the tumor cells for S-100 was observed; $>50 \%$ of the tumor cells were S100 positive $(+)$ in 15 of 17 cases and focal $(1+)$ and moderate $(2+)$ positivity was noted in the remaining 2 cases (Table 1). Next, immunocytochemistry for HMB45 was performed in 16 of the 17 cases in our cohort; in $13(81 \%)$ of these 16 cases, immunoreactivity of the tumor cells for HMB- 45 was observed. Greater than $50 \%$ of the tumor cells were HMB-45(+) in 6 cases. In 5 cases, between $10 \%$ and $50 \%$ of the tumor cells were HMB$45(+)$. In 2 cases, focal staining of the tumor cells $(<$ 10\%) was observed (Table 1, cases 7 and 16). Fifteen (88\%) of the 17 cases of metastatic melanoma exhibited immunoreactivity of the tumor cells for Mart-1. In 13 cases, $>50 \%$ of the tumor cells were immunoreactive for Mart-1. In 2 cases, $<50 \%$ of the tumor cells were immunoreactive for Mart-1 (Table 1, cases 1 and 6). Overall, immunoreactivity for all 3 melanoma markers was observed in 13 (76\%) of 17 cases of metastatic melanoma. One case was notable for the sparsity of tumor cells in the background of polymorphous lymphocytes (Table 1, case 2). Virtually all of the tumor cells were positive for $S-100$, HMB-45, and Mart-1 (Fig. 1). Two cases exhibited an S100(+)/HMB-45 negative (-)/Mart-1(-) immunophenotype and 1 case displayed an S-100(+)/HMB-45(-)/ Mart-1(+) immunoprofile (Fig. 1). Immunohistochemistry was also performed using cell block sections in 14 of 17 cases; the immunophenotype of the tumor cells was identical to that observed on the direct smears in all 14 cases. Immunohistochemistry could not be performed in the remaining 3 cases due to acellularity of the cell block preparations.

Next, we analyzed cases of metastatic melanoma, using cellular material derived from Diff-Quik-stained smears, for the presence or absence of mutations in BRAF. For this purpose, smears from 15 cases were retrieved from the archive and the coverslips removed. Subsequently, tumor-enriched areas were microdissected from each smear; the microdissected areas ranged from $24 \mathrm{~mm}^{2}$ to $56 \mathrm{~mm}^{2}$ (Fig. 2). The percentage tumor cellularity within these enriched areas was at least $70 \%$ for all cases and was $>90 \%$ in 11 of 15 cases (Table 2). DNA of high quality and purity was obtained in each case. Overall, a mutation in $B R A F$ was observed in $8(53 \%)$ of the 15 melanomas tested. Specifically, the V600E mutation was 


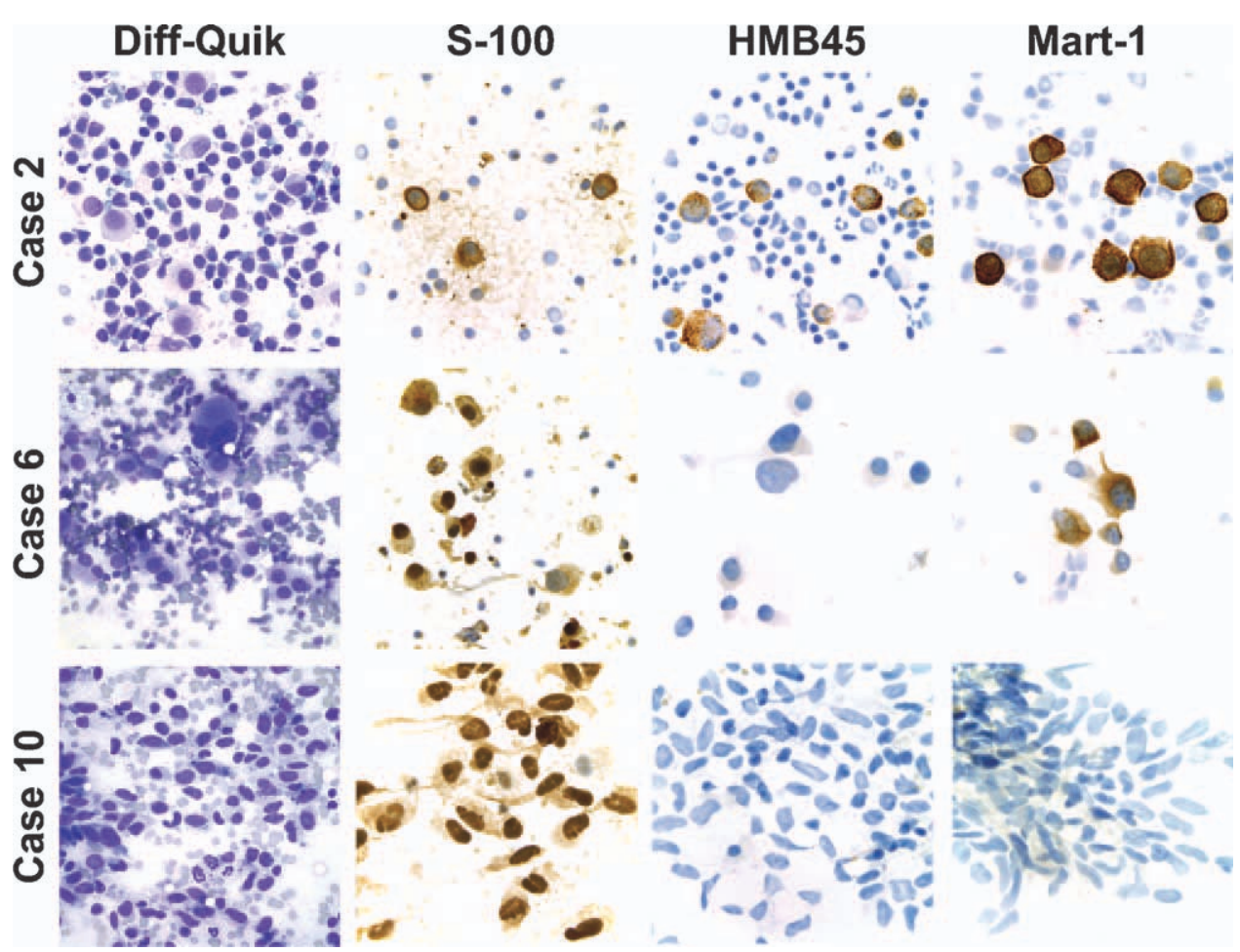

FIGURE 1. Immunocytochemistry is shown for S-100, HMB-45, and Mart-1 on direct smears in 3 cases of metastatic melanoma. The top row depicts a case in which the tumor cells are sparsely scattered in a background of lymphocytes. These cells are highlighted on all 3 immunocytochemical stains in contrast to the background lymphocytes, which are negative for all 3 markers. The middle and bottom rows illustrate cases of an S-100 positive (+)/HMB-45 negative (-)/Mart-1(+) and an S-100(+)/HMB-45(-)/ Mart-1(-) melanoma, respectively. The case numbers correspond to those listed in Table 1 (original magnification $\times 1000$ ).

detected in $5(33 \%)$ cases and the V600K mutation was detected in $3(20 \%)$ cases. For comparison, the corresponding cell blocks for each case were also tested in parallel. Seven, 3 , and 5 cell blocks exhibited sparse, moderate, and abundant cellularity, respectively; the percentage tumor cellularity varied from $<10 \%$ to $>90 \%$ (Table 2 ). Concordant sequencing results were observed in 14 (93\%) of 15 cases; however, in 1 case (Table 2, case 10), the mutation was not detected in the cellular material obtained from the cell block, whereas the V600E mutation was detected in the direct smear (Fig. 3). There were no cases in which a BRAF mutation was detected in the cell block material but not in the cellular material from the smears.

\section{DISCUSSION}

Difficulties can be encountered in the diagnosis of melanoma on FNA specimens owing to variations in cytomorphologic presentation and similarities to other malignant entities such as metastatic carcinoma and sarcoma. ${ }^{3-5,7-}$ ${ }^{11,19}$ An accurate cytodiagnosis allows for timely, appropriate staging of patients with this disease and the prompt formulation of appropriate next steps in management. ${ }^{6}$ In this regard, immunocytochemistry serves as an essential adjunct in the cytodiagnosis of melanoma. Furthermore, in this era of personalized medicine and evolving targeted chemotherapeutics, there is an increased need for molecular characterization of metastatic melanomas. Recently, this has been exemplified by the detection of activating $B R A F$ mutations in melanoma and subsequent ongoing clinical trials attempting to exploit this molecular signature from a targeted chemotherapeutic standpoint. ${ }^{2,15}$

Traditionally, cell blocks have represented the primary means by which additional cellular material is harvested for ancillary immunocytochemical and molecular diagnostic studies. Nonetheless, the absence of sufficient cellular material can pose a problem in the adequate workup of melanoma FNAs in some instances. The cell block cellularity is influenced by several variables 

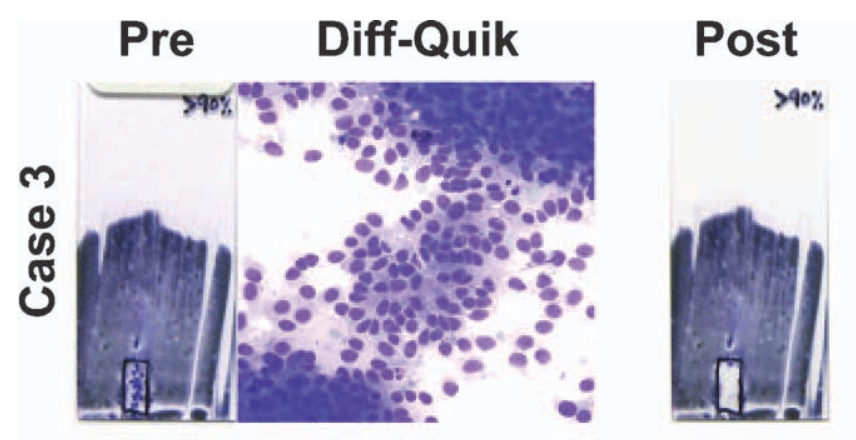

\section{Cell Block}
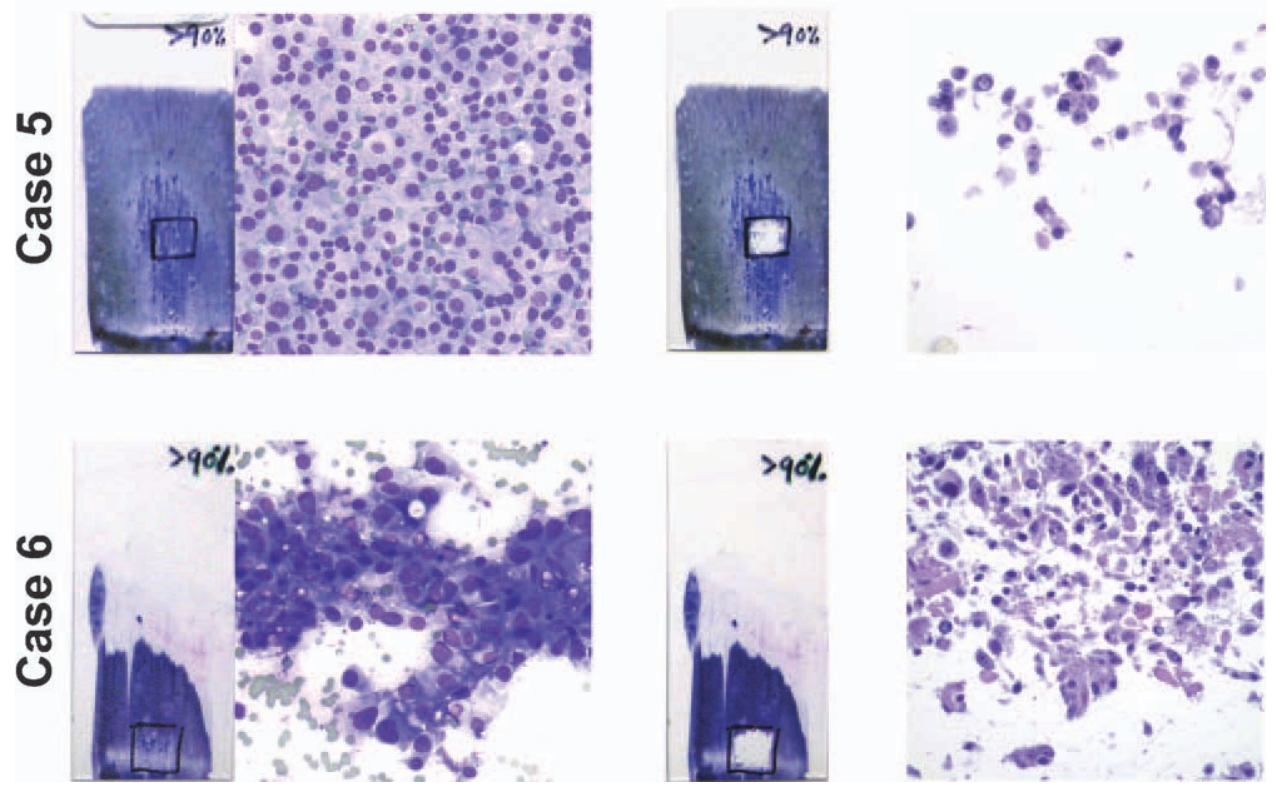

FIGURE 2. Cellular material used for BRAF mutational analysis is shown. Three representative cases corresponding to those listed in Table 2 are shown. The left column depicts the decoverslipped, Diff-Quik-stained direct smears before microdissection (Pre). Areas enriched in tumor cells were marked on the underside of the slides and the percentage of tumor cells present in each area is indicated on the upper right corner of each slide. Representative photomicrographs in each of the indicated areas are shown (original magnification $\times 600$ ). The middle column depicts the smears after microdissection (Post). Representative photomicrographs of the corresponding cell blocks are shown in the right column. The cell blocks in cases 3, 5, and 6 were of sparse, moderate, and abundant cellularity, respectively.

including the cellularity of the lesion being targeted by FNA, the percentage tumor cellularity in the lesion being sampled, the precision in targeting the lesion in successive needle passes, effective sampling of the lesion during dedicated FNA passes for the cell block, and postprocedural handling of the needle rinse specimen. In addition, the quantity of tumor cells in the cell block, as assessed by routine $\mathrm{H} \& \mathrm{E}$ staining of cell block sections, is not immediately known. Dedicated passes for the cell block may increase the probability that the cell block is of adequate tumor cellularity; however, it is not possible to ensure the effectiveness of additional cell block passes at the time of the FNA procedure. Recognizing the realistic possibility that cell blocks may provide inadequate material in scenarios in which the need for ancillary studies is the greatest, the aim of this study was 2-fold: 1 ) to confirm that diagnostic immunocytochemical stains for S-100, HMB45, and Mart-1 can be performed on direct aspirate smears successfully; and 2) to investigate the use of direct smears as a source of cellular material for the performance of $B R A F$ mutational analysis.

With regard to the first objective, we prospectively performed immunostains for S-100, HMB-45, and Mart1 in 17 consecutive FNAs of metastatic melanoma in 
Table 2. BRAF Mutational Analysis Using Direct Smears and Corresponding Cell Blocks of Metastatic Melanoma

\begin{tabular}{|c|c|c|c|c|c|c|c|c|}
\hline \multirow{2}{*}{$\begin{array}{l}\text { Case } \\
\text { No. }\end{array}$} & \multirow{2}{*}{$\begin{array}{l}\text { FNA } \\
\text { Site }\end{array}$} & \multicolumn{3}{|c|}{ Direct Smears } & \multicolumn{3}{|c|}{ Cell Blocks } & \multirow{2}{*}{$\begin{array}{l}\text { Direct } \\
\text { Smear/Cell } \\
\text { Block } \\
\text { Concordance }\end{array}$} \\
\hline & & $\begin{array}{l}\text { Microdissected } \\
\text { Area, } \mathrm{mm}^{2}\end{array}$ & $\begin{array}{l}\% \\
\text { Tumor }^{a}\end{array}$ & $\begin{array}{l}\text { BRAF } \\
\text { Mutation } \\
\text { Status }\end{array}$ & $\begin{array}{l}\text { Overall } \\
\text { Cellularity }\end{array}$ & $\begin{array}{l}\% \\
\text { Tumor }\end{array}$ & $\begin{array}{l}\text { BRAF } \\
\text { Mutation } \\
\text { Status }\end{array}$ & \\
\hline 1 & Right groin & 24 & $>90 \%$ & WT & $1+$ & $80 \%-90 \%$ & WT & Yes \\
\hline 2 & Left ear & 25 & $>90 \%$ & V600K & $1+$ & $>90 \%$ & V600K & Yes \\
\hline 3 & Left groin & 32 & $>90 \%$ & V600E & $1+$ & $<10 \%$ & V600E & Yes \\
\hline 4 & Left ear & 30 & $70 \%-80 \%$ & V600K & $2+$ & $30 \%-40 \%$ & V600K & Yes \\
\hline 5 & Left groin & 42 & $>90 \%$ & V600E & $2+$ & $80 \%-90 \%$ & V600E & Yes \\
\hline 6 & Left groin & 56 & $>90 \%$ & V600E & $3+$ & $>90 \%$ & V600E & Yes \\
\hline 7 & Left axilla & 32 & $>90 \%$ & V600K & $1+$ & $70 \%-80 \%$ & V600K & Yes \\
\hline 8 & $\begin{array}{l}\text { Right hilar } \\
\text { lymph node }\end{array}$ & 49 & $>90 \%$ & WT & $3+$ & $>90 \%$ & WT & Yes \\
\hline 9 & Right neck & 30 & $>90 \%$ & WT & $1+$ & $20 \%-30 \%$ & WT & Yes \\
\hline 10 & Right axilla & 25 & $80 \%-90 \%$ & V600E & $1+$ & $<10 \%$ & WT & No \\
\hline 11 & $\begin{array}{l}\text { Subcarinal } \\
\text { lymph node }\end{array}$ & 40 & $70 \%-80 \%$ & WT & $3+$ & $70 \%-80 \%$ & WT & Yes \\
\hline 12 & $\begin{array}{l}\text { Mediastinal } \\
\text { lymph node }\end{array}$ & 50 & $>90 \%$ & WT & $3+$ & $30 \%-40 \%$ & WT & Yes \\
\hline 13 & Right axilla & 40 & $>90 \%$ & V600E & $2+$ & $>90 \%$ & V600E & Yes \\
\hline 14 & Left arm & 49 & $>90 \%$ & WT & $1+$ & $80 \%-90 \%$ & WT & Yes \\
\hline 15 & Left neck & 50 & $80 \%-90 \%$ & WT & $3+$ & $20 \%-30 \%$ & WT & Yes \\
\hline
\end{tabular}

Abbreviations: FNA, fine-needle aspiration; WT, wild type.

${ }^{a}$ Percentage of tumor in the microdissected area on the direct smear

${ }^{b}$ Cellularity assessment: 0 , acellular; $1+$, sparse cellularity; $2+$, moderate cellularity; $3+$, abundant cellularity.

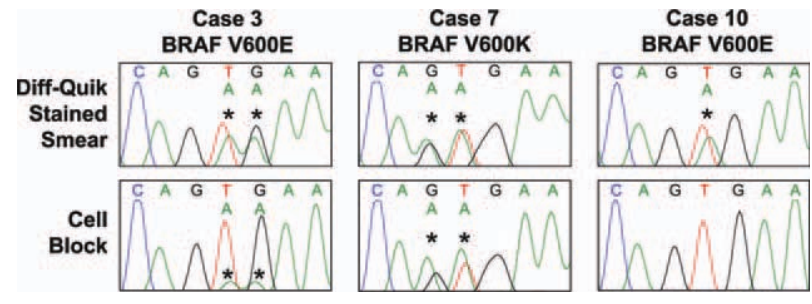

FIGURE 3. Representative sequencing results are shown for $B R A F$ mutations detected in direct smears and cell blocks. Three cases harboring BRAF mutations are shown. In cases 3 and 7 , the V6OOE and V6OOK mutations were detected, respectively, in DNA isolated from both the direct smears and cell blocks. In case 10, the V6O0E mutation was detected in the material obtained from the direct smear but not the cell block.

which unstained, air-dried direct smears were obtained on-site for immunocytochemistry. We demonstrated that S-100 was the most sensitive marker because all 17 cases of metastatic melanoma exhibited immunopositivity of the tumor cells for this marker. This is in concordance with previous reports that describe the high sensitivity of S-100 in detecting melanoma tumor cells. ${ }^{11,20}$ For exam- ple, Kapila et $\mathrm{al}^{11}$ examined 19 FNA cases of metastatic melanoma by S-100 immunocytochemistry and observed positivity for this marker in all cases. Nonetheless, because the specificity of S-100 for melanoma is lower compared with HMB-45 and Mart-1, and can label native lymph node elements such as sinus macrophages and dendritic cells, we also examined these latter 2 markers as well. ${ }^{20}$ We found that $81 \%$ and $88 \%$ of melanoma FNAs in our FNA cohort were positive for HMB-45 and Mart-1, respectively. These results are consistent with the findings by Zubovits et $\mathrm{al}^{20}$ that compared the relative sensitivities of these 3 markers. A few prior reports have demonstrated the utility of direct smears as a platform to perform immunocytochemical stains in the cytodiagnosis of melanoma; these studies used alcohol-fixed, Papanicolaou-stained smears that were destained before immunostaining. ${ }^{8,19}$ However, it has been noted that false-negative results could be observed in S-100 immunocytochemistry when alcohol-fixed smears are used. ${ }^{13,19}$ In the current study, air-dried direct smears were fixed in formalin before antigen retrieval and immunocytochemistry, thereby closely 
approximating the conditions of standard immunohistochemical procedures that use sections derived from formalin-fixed tissue. In 14 cases, tumor cells were present in the cell block, allowing for a comparison of immunophenotypes observed on immunostains performed using direct smears and formalin-fixed, paraffin-embedded cell block sections. Identical immunophenotypes were observed in each case, confirming that our protocol for performing immunocytochemistry on air-dried direct smears, after formalin fixation, closely approximates the conditions under which immunohistochemistry on cell block sections is performed. It is interesting to note that 1 FNA of metastatic melanoma exhibited a paucicellularity of tumor cells in a background of numerous polymorphous lymphocytes (Table 1, case 2). This scenario highlights a potential limitation of relying on cell blocks for immunocytochemical studies. Especially in this case, ensuring that the tumor cells would be adequately represented in the cell block during the on-site assessment of adequacy would be difficult, despite additional dedicated passes being performed. For this case, the immunostains for S-100, HMB-45, and Mart-1 selectively highlighted the sparsely scattered tumor cells in the background lymphoid milieu. Although not having performed S-100, HMB-45, and Mart-1 immunocytochemistry on nonmelanoma aspirates represents a limitation to the current study, our results demonstrate the diagnostic utility of performing immunocytochemistry for these 3 markers in the diagnosis of melanoma FNAs.

Another key finding in the current study is that the use of cytologic direct smears proved to be a robust and reliable methodology for molecular testing. Mutation testing was successfully performed using cellular material microdissected from archived, Diff-Quik-stained, decoverslipped smears. Overall, BRAF mutations were observed at the expected frequency ${ }^{15}$ because $53 \%$ of the tumors analyzed in this study harbored mutations in $B R A F$. The majority of the mutations resulted in the V600E substitution and a minority of tumors exhibited the $\mathrm{V} 600 \mathrm{~K}$ substitution. This is consistent with prior observations that the most common $B R A F$ mutation in melanoma is V600E followed by V600K. ${ }^{16,17}$ In all but 1 case, BRAF sequencing results using cellular material obtained from the direct smears yielded results that were concordant with those obtained from the corresponding cell blocks. Of note, the 1 discordant case in which a
$B R A F$ mutation was identified in the direct smear but not the corresponding cell block was due to limited tumor cellularity in the latter. Specifically, the overall cellularity of the cell block was low, and $>90 \%$ of the cells present in the cell block were comprised of background lymphocytes (Table 2, case 10). Given the limited ability to microdissect for tumor cells in cell blocks, this case highlights a potential limitation of cell blocks containing tumor cells dispersed among background-contaminating benign cells. Moreover, because cell blocks represent a cumulative, pooled specimen derived from multiple needle passes, dilution of the tumor cell population by benign cellular elements can yield false-negative mutation results due to the limited analytic sensitivity of many molecular tests. The advantages of using air-dried, Diff-Quik-stained smears for molecular testing include the higher quality of DNA isolated relative to that prepared from formalinfixed tissue and the long-term stability of the DNA in archived smears. ${ }^{21}$ Most importantly, the Diff-Quik staining method is quick, inexpensive, and allows for the rapid verification of tumor cell adequacy. Tumor-enriched areas can be easily identified and marked before triaging for molecular studies. In this regard, our study differs from a recent study by Sviatoha et al who performed BRAF mutational analysis on melanoma FNAs. ${ }^{22}$ In their study, pelleted, frozen cellular material from aspirates was used for molecular testing. In this setting, it is not possible to enrich for the tumor cell population; hence, if the representation of the tumor cell population is low in a particular aspirate, a false-negative result may be obtained on molecular assays designed to detect mutations. Recently, we observed that air-dried smears represented a robust source of cellular material for epidermal growth factor receptor $(E G F R)$ and KRAS mutational analysis of pulmonary nonsmall cell lung cancer. ${ }^{23}$ Our success in performing $B R A F$ mutational analysis in this study reiterates the utility and effectiveness of direct smears for molecular assays. Overall, these findings are fortuitous for cytopathologists who are responsible for ensuring immediate adequacy assessments and for triaging cellular material based on examining DiffQuik-stained smears on-site.

In the current study, we used decoverslipped smears as a source of cellular material for molecular analysis. Although the Diff-Quik staining protocol and the process of decoverslipping archival smears do not compromise the quality of the DNA isolated for molecular studies, as 
evidenced by our findings, we realize that the procedure of removing coverslips can still be time-consuming. Therefore, to ensure a quicker turnaround time for obtaining results of mutational analysis, it would be more convenient for the cytopathologist to anticipate that additional cellular material will be needed for molecular analysis and to maintain at least 1 air-dried, uncoverslipped slide at the time of the FNA procedure. Should molecular testing be requested, this slide can be promptly stained with Diff-Quik, marked to indicate tumor-enriched areas, and immediately sent to the molecular diagnostics laboratory for mutational analysis.

The current study findings have important implications for the optimal triage of FNA speciemens for cytodiagnosis and molecular analysis. Preparing multiple unstained direct smears from 1 or more FNA passes would allow the cytologist to confirm on-site that adequate material has been obtained not only for cytodiagnosis but also for ancillary studies. Specifically, in addition to the routinely prepared air-dried, Diff-Quikstained and alcohol-fixed, Papanicolaou-stained smears per pass, 1 or more additional smears could be prepared for ancillary studies by evenly distributing the aspirate material over multiple slides. Visualization of tumor cells on the Diff-Quik-stained smear would signify the presence of tumor cells in the additional unstained direct smears. We acknowledge that the continued use of needle rinses and dedicated passes for the cell block would serve to preserve the remainder of the cellular material in formalinfixed, paraffin-embedded form for future studies as needed. This protocol, however, would reduce and potentially eliminate the reliance solely on the cell block as a source of cellular material for these studies. Thus, optimization of FNA specimen triage during on-site assessments can further cement the essential role of FNA with cytologic examination in the management of patients with metastatic melanoma. In an era in which the number of necessary ancillary studies is likely to increase, cytopathologists can meet the challenge of optimally using limited FNA material and simultaneously preventing additional invasive procedures that could result from scenarios in which the cell blocks exhibit insufficient cellularity.

\section{FUNDING SUPPORT}

No specific funding was disclosed.

\section{CONFLICT OF INTEREST DISCLOSURES}

The authors made no disclosures.

\section{REFERENCES}

1. Jemal A, Siegel R, Xu J, Ward E. Cancer statistics, 2010. CA Cancer J Clin. 2010;60:277-300.

2. Flaherty KT, Puzanov I, Kim KB, et al. Inhibition of mutated, activated BRAF in metastatic melanoma. $N$ Engl $J$ Med. 2010;363:809-819.

3. Murali R, Doubrovsky A, Watson GF, et al. Diagnosis of metastatic melanoma by fine-needle biopsy: analysis of 2,204 cases. Am J Clin Pathol. 2007;127:385-397.

4. Cangiarella J, Symmans WF, Shapiro RL, et al. Aspiration biopsy and the clinical management of patients with malignant melanoma and palpable regional lymph nodes. Cancer. 2000;90:162-166.

5. Murali R, Loughman NT, McKenzie PR, Watson GF, Thompson JF, Scolyer RA. Cytologic features of metastatic and recurrent melanoma in patients with primary cutaneous desmoplastic melanoma. Am J Clin Pathol. 2008;130:715-723.

6. Murali R, Thompson JF, Uren RF, Scolyer RA. Fine-needle biopsy of metastatic melanoma: clinical use and new applications. Lancet Oncol. 2010;11:391-400.

7. Elliott D, Pitman MB. Malignant melanoma with a myxoid stroma: a diagnostic pitfall on fine-needle aspiration biopsy. Diagn Cytopathol. 2001;25:185-190.

8. Lai R, Redburn J, Nguyen GK. Cytodiagnosis of metastatic amelanotic melanomas by fine-needle aspiration biopsy: adjunctival value of immunocytochemistry and electron microscopy. Cancer. 1998;84:92-97.

9. Saqi A, McGrath CM, Skovronsky D, Yu GH. Cytomorphologic features of fine-needle aspiration of metastatic and recurrent melanoma. Diagn Cytopathol. 2002;27:286-290.

10. Fetsch PA, Marincola FM, Filie A, Hijazi YM, Kleiner DE, Abati A. Melanoma-associated antigen recognized by $\mathrm{T}$ cells (MART-1): the advent of a preferred immunocytochemical antibody for the diagnosis of metastatic malignant melanoma with fine-needle aspiration. Cancer. 1999;87:37-42.

11. Kapila K, Kharbanda K, Verma K. Cytomorphology of metastatic melanoma- use of S-100 protein in the diagnosis of amelanotic melanoma. Cytopathology. 1991;2:229-237.

12. Pelosi G, Bonetti F, Colombari R, Bonzanini M, Iannucci A. Use of monoclonal antibody HMB-45 for detecting malignant melanoma cells in fine needle aspiration biopsy samples. Acta Cytol. 1990;34:460-462.

13. Simmons TJ, Martin SE. Fine-needle aspiration biopsy of malignant melanoma: a cytologic and immunocytochemical analysis. Diagn Cytopathol. 1991;7:380-386.

14. Arkenau HT, Kefford R, Long GV. Targeting BRAF for patients with melanoma. Br J Cancer. 2010;104:392-398.

15. Flaherty KT. Narrative review: BRAF opens the door for therapeutic advances in melanoma. Ann Intern Med. 2010;153:587-591. 
16. Long GV, Menzies AM, Nagrial AM, et al. Prognostic and clinicopathologic associations of oncogenic BRAF in metastatic melanoma. J Clin Oncol. 2011;29:1239-1246.

17. Rubinstein JC, Sznol M, Pavlick AC, et al. Incidence of the V600K mutation among melanoma patients with BRAF mutations, and the potential therapeutic response to the specific BRAF inhibitor PLX4032. J Transl Med. 2010;8:67.

18. Roh MH, Schmidt L, Placido J, et al. The application and diagnostic utility of immunocytochemistry on direct smears in the diagnosis of pulmonary adenocarcinoma and squamous cell carcinoma [published online ahead of print April 15, 2011]. Diagn Cytopathol. doi: 10.1002/dc.21680.

19. Piao Y, Guo M, Gong Y. Diagnostic challenges of metastatic spindle cell melanoma on fine-needle aspiration specimens. Cancer. 2008;114:94-101.
20. Zubovits J, Buzney E, Yu L, Duncan LM. HMB-45, S100, NK1/C3, and MART-1 in metastatic melanoma. Hum Pathol. 2004;35:217-223.

21. Killian JK, Walker RL, Suuriniemi M, et al. Archival fineneedle aspiration cytopathology (FNAC) samples: untapped resource for clinical molecular profiling. J Mol Diagn. 2010;12:739-745.

22. Sviatoha V, Tani E, Ghaderi M, Kleina R, Skoog L. Assessment of V600E mutation of BRAF gene and rate of cell proliferation using fine-needle aspirates from metastatic melanomas. Anticancer Res. 2010;30:3267-3272.

23. Betz BL, Roh MH, Weigelin HC, et al. The application of molecular diagnostic studies interrogating EGFR and KRAS mutations to stained cytologic smears of lung carcinoma. Am J Clin Pathol. In press. 